\title{
Research on dynamic responses of incompletely water- filled cylindrical shell subjected to explosion impact
}

\author{
Fuyin Gao ${ }^{1}$, Chong $\mathrm{Ji}^{2}$, Ying $\mathrm{Liu}^{3}$, Liangyu Cheng ${ }^{4}$, Yang $\mathrm{Yu}^{5}$ \\ College of Field Engineering, The Army Engineering University of PLA, Nanjing, 210007, China \\ ${ }^{2}$ Corresponding author \\ E-mail: 1472564948@qq.com, ${ }^{2} 2468645816 @ q q . c o m,{ }^{3} 465718256 @ q q . c o m,{ }^{4} c l y c h m y @ q q . c o m$, \\ 5962823438@qq.com
}

Received 11 September 2018; accepted 10 October 2018 DOI https://doi.org/10.21595/vp.2018.20215

Check for updates

Copyright $(C) 2018$ Fuyin Gao, et al. This is an open access article distributed under the Creative Commons Attribution License, which permits unrestricted use, distribution, and reproduction in any medium, provided the original work is properly cited.

\begin{abstract}
Numerical simulation on the deformations and damages of incompletely water-filled cylindrical shells subjected to explosion impacts were carried out. The Research shows that the degree of deformation and failure of the incompletely liquid-filled cylindrical shell is smaller than that of the empty cylindrical shell in the same condition. The dynamic response of the completely water-filled cylindrical shell subjected to $75 \mathrm{gTNT}$ explosion impact is the same with the one of partially water-filled cylindrical shell. The anti-explosion performance of the $95 \%$ volume water-filled cylindrical shell subjected to $200 \mathrm{gTNT}$ explosion impact is better. The explosion direction greatly influenced the dynamic response of partially filled-water cylindrical shell. The dynamic response of a partially water-filled cylindrical shell is approximately the same as that of the fully water-filled cylindrical shell, when the explosion direction is on one side of the water. The results show that internal pressure of water will increase when subjecting to impact loading, the anti-blast ability of tube structure significantly enhanced.
\end{abstract}

Keywords: mechanics of explosion, explosion impact, fluid-structure interaction, incompletely water-filled cylindrical shell, numerical simulation.

\section{Introduction}

Oil and gas pipeline are conducive to the quick and efficient transportation of oil and gas products, which can meet the needs of different regions and consumption levels, and provide a guarantee for the strategic deployment of national energy security. Once the oil and gas pipeline damaged, it will cause huge economic losses. Therefore, we must make clear the dynamic behavior of oil and gas pipelines subjected to explosion impact loading. Previous studies have focused on the dynamic response of thin-walled cylindrical shells subjected to explosive shock loading, like W. J. Schuman Jr [1], Chung Kim Yuen [2] et al., but few studies have focused on the deformation and failure of liquid-filled cylindrical shells. The liquid-filled medium in the pipeline can absorb part of the explosive shock energy, which can hinder the deformation of the pipeline. Nishida M. et al. [3] investigated that the diameter of the plug of the water-filled tube was slightly larger than one of the empty tube. But Qasim H. Shah et al. [4] considered that deformations of the water-filled pipes were smaller, compared with deformations of the empty pipes under impacts. Finite element method can solve the dynamic response of cylindrical shell structure by Prinja Nawal K. [5], it can analyze effectively the deformation and failure of water-filled cylindrical shell.

In the present paper, the numerical simulation on incompletely water-filled cylindrical shells of $2.75 \mathrm{~mm}$ thickness subjected to explosion impact by $75 \mathrm{gTNT}$ charge and 200gTNT charge with different stand-off distances were carried out. It focused mainly on the anti-explosion performance of the filling medium (water), TNT charge mass. 


\section{Numerical simulation}

\subsection{The calculation model}

The calculation model involves in water-filled cylindrical shell, TNT charge and air. The model of metallic cylindrical shell adopted the purely Lagrangian, others adopted ALE. The mesh size of the air, water, TNT charge and stent is $0.25 \mathrm{~cm}$. The mesh size of the metal cylindrical shell from midpoint to $10 \mathrm{~cm}$ away from midpoint is $0.125 \mathrm{~cm}$, the other of which is $0.5 \mathrm{~cm}$. A quarter of model was established, which adopted SOLID164 solid element and g-cm- $\mu$ s unit, as showed in Fig. 1.

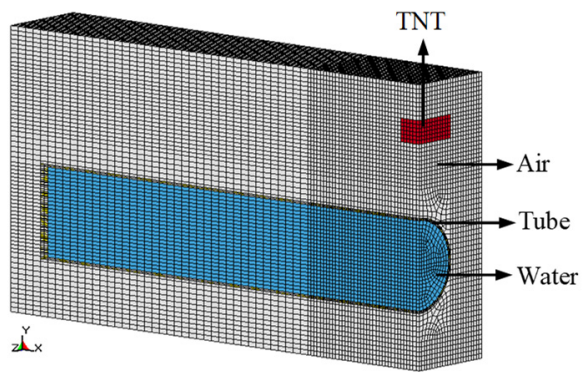

Fig. 1. The calculation model

\subsection{The material parameter}

The TNT model used Jones-Wilkens-Lee (JWL) equation of state (EOS) models, which engineering calculations is:

$p=\frac{\rho_{0} c^{2} \mu\left[1+\left(1-\gamma_{0} / 2\right) \mu-a \mu^{2} / 2\right]}{\left[1-\left(S_{1}-1\right) \mu-S_{2} \mu^{2} /(\mu+1)-S_{3} \mu^{3} /(\mu+1)^{2}\right]}+\left(\gamma_{0}+\alpha \mu\right) E$.

The state parameters are: $\rho_{e}=1.63 \mathrm{~g} / \mathrm{cm}^{3}, D=6.93 \mathrm{~km} / \mathrm{s}, p_{C J}=21.1 \mathrm{GPa}, A_{1}=371.1 \mathrm{GPa}$, $B_{1}=3.230 \mathrm{GPa}, R_{1}=4.16, R_{2}=0.96, \omega=0.30, E_{0}=7.0 \times 10^{9} \mathrm{~J} / \mathrm{m}^{3}$.

The air modeled used the simplest forms of EOS. The expression of pressure is:

$p=(\gamma-1) \rho e$,

where the air density $\rho$ is $1.225 \mathrm{~kg} / \mathrm{m}^{3}, \gamma=1.4, e=2.068 \times 10^{5} \mathrm{~kJ} / \mathrm{kg}$.

The water model adopted *MAT_NULL*. Where $\rho_{0}=1000.0 \mathrm{~kg} / \mathrm{m}^{3}, \gamma_{0}=0.50$.

The tube steel model adopted the Johnson -Cook material model [23], it defines the $\sigma_{y}$ is:

$\sigma_{y}=\left[A+B\left(\bar{\varepsilon}^{p}\right)^{n}\right]\left[1+C \ln \dot{\varepsilon}^{*}\right]\left[1-(T *)^{m}\right]$.

The expression of effective fracture strain defined by:

$\varepsilon_{f}-\left[D_{1}+D_{2} \exp D_{3} \sigma^{*}\right]\left[1+D_{4} \ln \dot{\varepsilon}^{*}\right]\left[1+D_{5} T^{*}\right]$

The material constants of the typical metal cylindrical shell are: $\rho=7850 \mathrm{~kg} / \mathrm{m}^{3}, \mu=0.3$, $E_{p}=210 \mathrm{GPa}, A=560 \mathrm{MPa}, B=560 \mathrm{MPa}, n=0.48$.

\section{Numerical analysis}

The ALE fluid-solid coupling model has shown that it is possible to analyze the dynamic response of fully filled-water cylindrical shells subjected to blast loading, but it does not consider 
the deformation and damage of partially filled-water cylindrical shells. In fact, a liquid-filled pipe subjected to an explosion loading may also be not filled full water, because of the presence of the air inside the tube. The part-liquid cylindrical shell will show some different characteristics, and the air inside the tube provides the movement space for the liquid in the pipe. The dynamic response of partially filled cylindrical shells subjected to explosion impact will be different compared with that of full charging pipe.

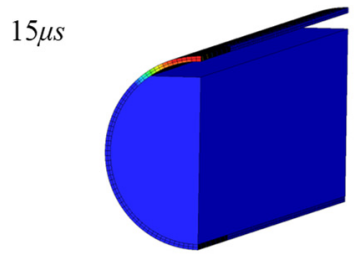

a)

$100 \mu \mathrm{s}$

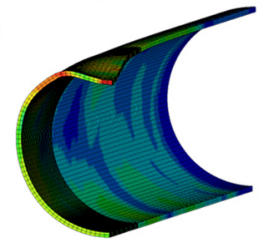

d)

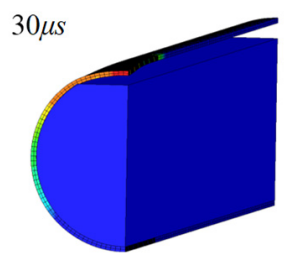

b)

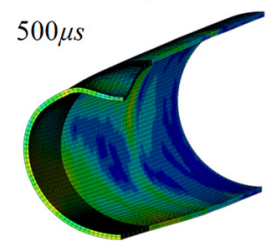

e)

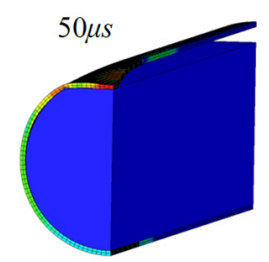

c)

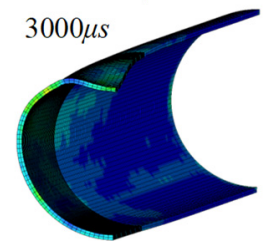

f)

Fig. 2. The deformation process of the $2.75 \mathrm{~mm}$ thickness water-filled cylindrical shell under the $95 \%$ filling volume subjected to $75 \mathrm{gTNT}$ charge explosion impacting

$55 \mu \mathrm{s}$

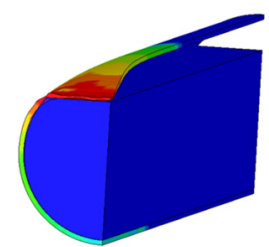

a)

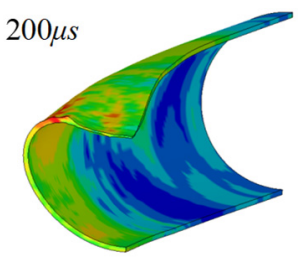

b)

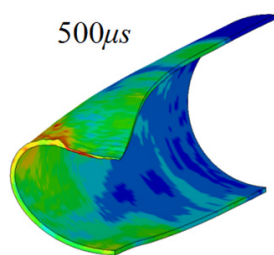

c)

Fig. 3. The deformation process of the $2.75 \mathrm{~mm}$ thickness water-filled cylindrical shell under the $85 \%$ filling volume subjected to $200 \mathrm{gTNT}$ charge explosion impacting

Fig. 2 is the deformation process of the $2.75 \mathrm{~mm}$ thickness water-filled cylindrical shell under the $95 \%$ filling volume subjected to explosion impact of $75 \mathrm{gTNT}$ charge. Different from the fully water-filled cylindrical shell, the first interaction between the shell and the shell at $30 \mathrm{~s}$ is both sides of the horizontal plane. At the time of $50 \mathrm{~s}$, the central point of the explosion surface is collided with the water medium, and the plastic hinge formed on both sides of the surface of the water medium plane. At $100 \mathrm{~s}$, the sunken area continues to deform along the plastic hinge, the plastic hinge is prominent, and the shell strain changes. The maximum strain near the center of the explosion surface reaches $42.2 \%$. At the same conditions, the forming time of plastic hinge of empty tube is later, and is much smooth, and it will not form a convex shape. The maximum strain near the center point of the explosive surface is only $19.2 \%$. The deformation of the cylindrical shell structure is stable at $500 \mathrm{~s}$, and the depth of the sunken pit is smaller than that of the empty tube, but the radial deformation area is larger. As shown in Fig. 3, when the distance between $200 \mathrm{gTNT}$ and $9 \mathrm{~cm}$ is $55 \mathrm{~s}$, the center of the explosion surface will collide with the water medium. At this time, the two sides contacting with the water medium plane also form plastic hinge and form a sharper convex shape. The highly speed collision between the shell and the water medium, which is coupled with the water medium Poisson, together to absorb the blast impact energy. Due to the continuous interaction with the water medium, the strain in the sunken area increases gradually, and the shell near the center of the blasting surface becomes very thin. The 
maximum strain even reaches $69 \%$, which is far beyond the limit rupture strain of the steel itself. It can be seen that the ultimate fracture strain of the cylindrical shell is increased by the waterfilled medium under the blast impact, and the impact toughness and strength of the cylindrical shell structure are improved.

The pressure values of the center point of partially filled cylindrical shells subjected to explosion impact show the regular trend, and the peak pressure peak of the center point is as shown in Fig. 4. After impacted by the shell, the peak of the secondary maximum pressure of the water medium is not the center point, but the water-filled volume is $95 \%$, and then the pressure peak does not fluctuate greatly. The larger end pressure peaks are $100 \%$ water filled and $95 \%$ water filled. It has intense pressure oscillation before $1000 \mu \mathrm{s}$ in the end of $100 \%$ water-filled cylindrical shell, after $1000 \mu \mathrm{s}$, the end of $95 \%$ water-filled cylindrical shell start oscillation to $2000 \mu \mathrm{s}$. The end deformation is not consistent, because water shock pressure small end deformation is broadly in line with a final degree of transverse convex shape. The center peak pressure is $2.47 \mathrm{MPa}$ under $200 \mathrm{gTNT}$ explosion and distance of $9 \mathrm{~cm}$, but the end of the peak pressure in the $95 \%$ and $85 \%$ water-filled volume is more than $100 \%$ water-filled volume. The pressure peak is in the $95 \%$ water-filled volume, indicated strong oscillations and large deformation of the cylindrical shell, in the condition of the water-filled cylindrical shell water medium was more intense, the end deformation was severe.

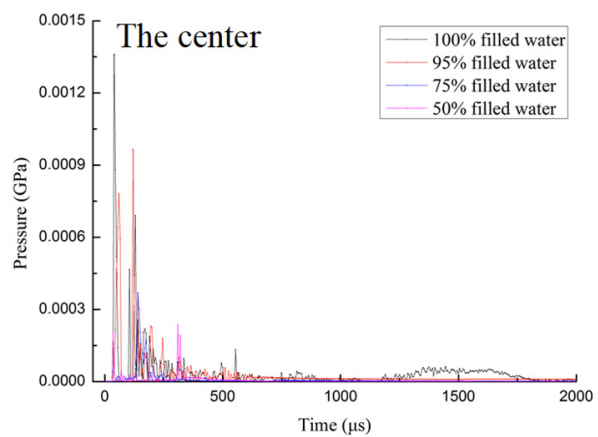

a)

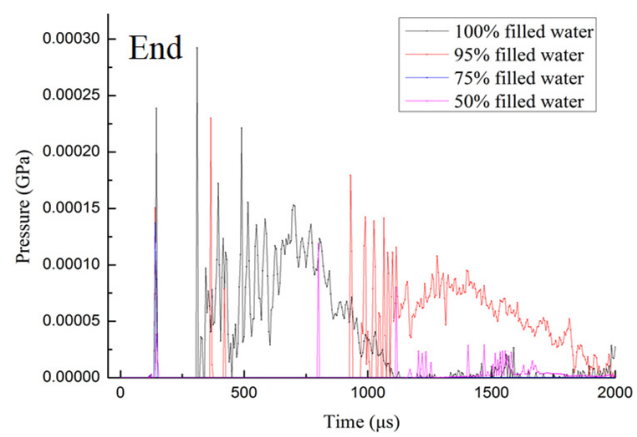

b)

Fig. 4. The center and end pressure curve of a water filled cylindrical shell subjected to the $75 \mathrm{gTNT}$ explosion impact and $5 \mathrm{~cm}$ explosion distance

Fig. 6 is the radial and axial deformation curve of a water-filled cylindrical shell subjected to the $75 \mathrm{gTNT}$ charge and $5 \mathrm{~cm}$ explosion distance. The axial and radial deformation curves of the incomplete cylindrical shell are consistent with the trend from the figure, namely water filled more sunken, deformation is smaller. The sunken depth of the water $50 \%$ filled volume is similar to the depth of empty tube, which is $5.7 \mathrm{~cm}$ and $5.78 \mathrm{~cm}$ respectively. The sunken curvature radius of the empty tube is only larger. It indicates that $75 \mathrm{gTNT}$ has no detonation effect in the $50 \%$ volume under the $5 \mathrm{~cm}$ explosive distance. When the volume of water-filled reaches $95 \%$ and $100 \%$, the radial curve of $100 \%$ water-filled volume is larger when $95 \%$ water-filled volume. Due to the intense collision between the water and the shell, the maximum strain near the center of the explosion surface reaches $42.2 \%$ when the water-filled volume is $95 \%$, and the maximum strain is only $5.5 \%$ when it is $100 \%$, the shell and the water become the whole movement.

As mentioned above, the empty tube subjected to $200 \mathrm{gTNT}$ explosion impact and $9 \mathrm{~cm}$ explosion distance will break through rupture; but when the water-filled volume reaches $75 \%$, the water-filled cylindrical shell will only have depression deformation and overall bending, as shown in Fig. 7. Compared with $75 \mathrm{gTNT}$ explosion impact, the sunken deformation degree is more serious, and the deformation regularity is different. When the water filling volume is $95 \%$, the sunken depth is the smallest, and the axial and radial deformation curves are uneven. It shows the violent collision degree between the shell and the water, resulting in the maximum strain of the shell is $57.3 \%$. When the water filling volume is $85 \%$, the plastic hinge appears near the center 
point of $3 \mathrm{~cm}$, and is obviously convex and the axial deformation curve is not smooth. This is due to the collision between the shell and the water, and the maximum strain of the shell is $68.7 \%$. The axial and radial deformation curves of the $75 \%$ and $100 \%$ water-filled volumes tube are smoother, and the impact of the shells on the water is relatively low. The maximum strain of $75 \%$ falls to $65 \%$, while the $100 \%$ water-filled volume is only $13.4 \%$, the axial deformation area is the smallest.

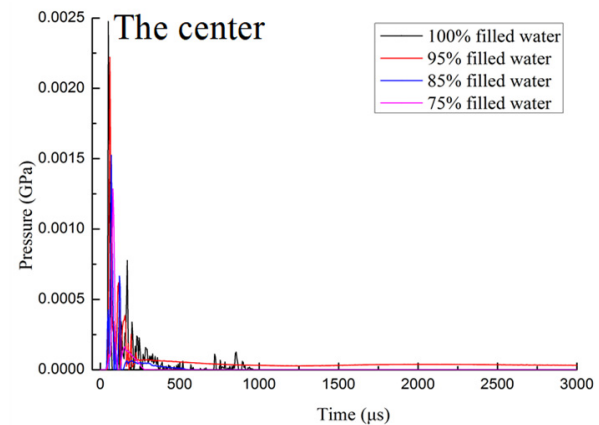

a)

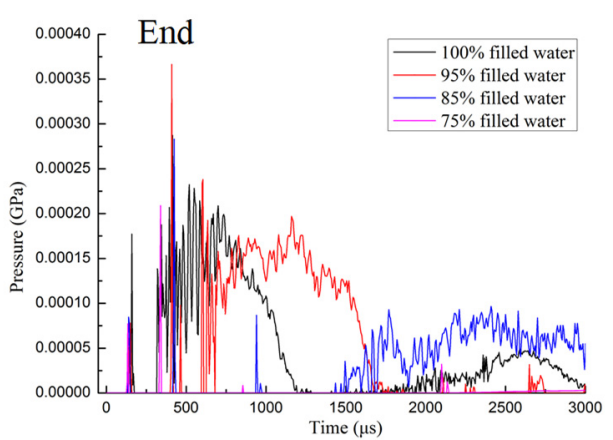

b)

Fig. 5. The center and end pressure curve of a water filled cylindrical shell subjected to the $200 \mathrm{gTNT}$ explosion impact and $9 \mathrm{~cm}$ explosion distance

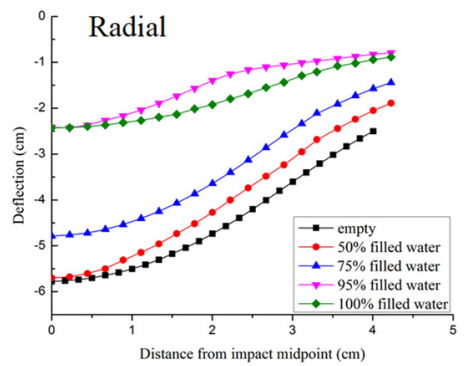

a)

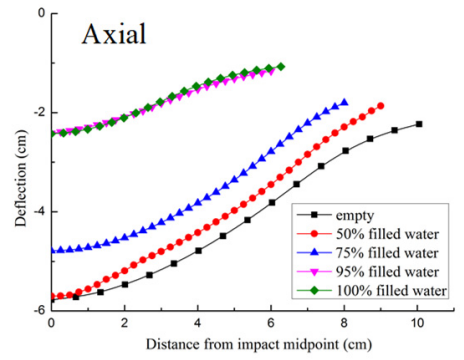

b)

Fig. 6. The radial and axial deformation curve of a water filled cylindrical shell subjected to the $75 \mathrm{gTNT}$ explosion impact and $5 \mathrm{~cm}$ explosion distance

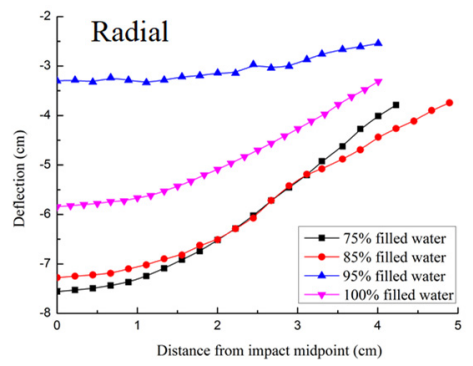

a)

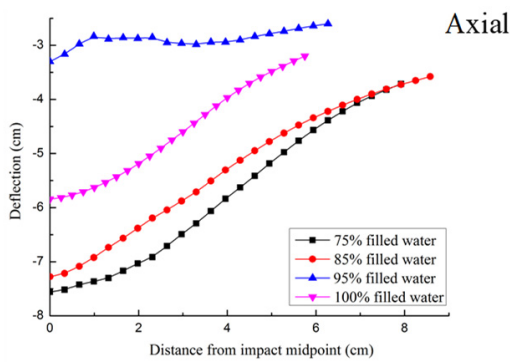

b)

Fig. 7. The radial and axial deformation curve of a water filled cylindrical shell subjected to the 200gTNT explosion impact and $9 \mathrm{~cm}$ explosion distance

Except the water-filled volume affects antiknock performance of cylindrical shell, the explosion source orientation on anti-explosion performance of the incomplete water-filled cylindrical shell will also have an impact. Fig. 8 is the radial and axial deformation curve of a water filled cylindrical shell subjected to the $75 \mathrm{gTNT}$ front and inferior explosion impact and $5 \mathrm{~cm}$ explosion distance. It can be seen that the depression of the cylindrical shell under inferior explosion impact is smaller and close to the depressions of the fully water filled cylindrical shells. 
Therefore, Studying the antiknock performance of incomplete water filled cylindrical shells (water volume is greater than $50 \%$ ), if the explosion occurs inferior of partial water-filled cylindrical shells, it can be equivalent to the dynamic response of cylindrical shells with $100 \%$ water-filled volume subjected to the impact of explosion.

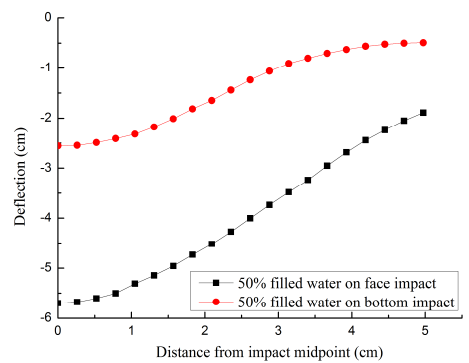

a)

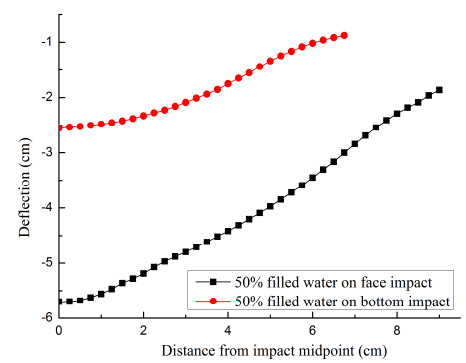

b)

Fig. 8. The radial and axial deformation curve of a water-filled cylindrical shell subjected to the $75 \mathrm{gTNT}$ front and inferior explosion impact and $5 \mathrm{~cm}$ explosion distance

\section{Conclusions}

1) The pressure of the water increased by the joint action of the explosion impact and the deformation of the shell, which causes the Poisson coupling between the water and the cylindrical shell. Because of the presence of air in the pipe, when the impact is on the cylindrical shell, the air in the pipe provides the movement space for the liquid in the pipe. The degree of deformation and failure of the incompletely filled-water cylindrical shell is smaller than that of the empty pipe in the same condition;

2) The dynamic response of the fully water-filled cylindrical shell subjected to $75 \mathrm{gTNT}$ explosion impact is the same as that of incompletely water-filled cylindrical shell. The anti-explosion performance of the $95 \%$ volume water-filled cylindrical shell subjected to 200gTNT explosion impact is better. The explosion direction greatly influenced the dynamic response of partially filled-water cylindrical shell. The dynamic response of the incompletely water-filled cylindrical shell is approximately the same as that of the fully water-filled cylindrical shell when the explosion direction is on one side of the water.

\section{Acknowledgements}

This research was financially supported by the National Nature Science Foundation of China, Nos. 51678567, 11102233 and 51608530.

\section{References}

[1] Schuman Jr W. J. A Failure Criterion for Blast Loaded Cylindrical Shells. Aberdeen Proving Ground, Maryland, U. S. Army Ballistic Research Laboratories, Report No. 1292, 1965, p. 1-128.

[2] Chung Kim Yuen S., Nurick G. N., Brinckmann H. B., Blakemore D. Response of cylindrical shells to lateral blast load. International Journal of Protective Structures, Vol. 4, 2013, p. 209-230.

[3] Nishida M., Tanaka K., Ito M. Deformation and perforation of water-filled and empty aluminum tubes by a spherical steel projectile: experimental study. Impact Engineering and Application, 2001, p. 375-380.

[4] Qasim H. Shah Experimental and numerical study on the orthogonal and oblique impact on water filled pipes. International Journal of Impact Engineering, Vol. 38, 2011, p. 330-338.

[5] Prinja Nawal K. Combined beam element for large dynamic motion of whipping pipes with fluid structure inter-action. Finite Element in Analysis and Design, Vol. 11, 1992, p. 117-152. 